\title{
Research on the Methods of Holding Control Strategy for Bus
}

\author{
Tingting $\mathrm{Hu}^{1, \mathrm{a}^{*}}$, Xiucheng Guo ${ }^{2, \mathrm{~b}}$, Zhenping $\mathrm{Xi}^{3, \mathrm{c}}$ and Jiabin $\mathrm{Li}^{4, \mathrm{~d}}$ \\ ${ }^{1}$ School of Transportation, Southeast University, Nanjing, Jiangsu, China \\ ${ }^{2}$ School of Transportation, Southeast University, Nanjing, Jiangsu, China \\ ${ }^{3}$ School of Transportation, Southeast University, Nanjing, Jiangsu, China \\ ${ }^{4}$ Hangzhou City Planning and Design Academy, Hangzhou, Zhejiang, China \\ ahttazseu@foxmail.com, bseuguo@163.com, ctpxzp@126.com, ${ }^{\mathrm{d}}$ nbamagic@163.com
}

\section{Keywords: Bus; Holding Control; Dynamic Framework; Holding Time Threshold}

Abstract. Research on the holding control methods for bus can improve the reliability of bus transfer and reduce travel delays of passengers effectively. What's more, it can help to improve the quality and attractiveness of public transport service and promote the priority development of urban public transport. This paper proposes a dynamic holding control strategy. And by analyzing the adaptability of holding control from the aspect of Interval and arrival punctuality of vehicle, the appropriate line types is determined. And a dynamic framework is created. Then, synthetically considering factors such as cost of enterprise, passengers and capacity constraints, the model of holding control is established, which is aiming at the minimum total cost and to calculate the holding time threshold. An example is given so as to analyze the effectiveness of holding control.

\section{Introduction}

The unreliability and long waiting time for transfer are the key factors for the low-quality and weak-attractive services of ground bus. The operation of ground buses is easily affected by the factors such as road conditions and emergencies, which usually results in delays. In this condition, passengers who have to transfer to finish a trip would be faced with transfer inconvenience like long waiting time. In 2011, the average travel time of bus in the central city of Nanjing is 45.5 minutes, and the average waiting time of transfer is 5.3 minutes, which ac-counts for $11.6 \%$ of the average travel time.

Dynamic coordinated control can effectively guarantee the quality of public transport service, and the holding control is one of the most important strategies. Through implementing the holding control at the transfer stations, punctual buses are controlled to wait for late buses which have transfer passengers among them, improving the probability of successful transfer and reducing the travel delay caused by transfer failures. However, the holding control would increase the waiting time of passengers who don't need transfer. Therefore, changes in the bus system cost due to the holding control need to be studied so that the decision of holding control or not can be made aiming at minimizing the system cost.

\section{Frame work of Holding Control}

Dynamic holding control strategy. A dynamic holding control strategy is proposed and the model of holding control is established to calculate the holding time threshold, which takes the enterprises, passengers and vehicles into consideration. It is assumed that the arrival time of delayed vehicles can be predicted. If the predicted arrival time of delayed vehicles is beyond the holding time thresh-old, the holding control will not be carried out. If the predicted arrival time is within the threshold, the holding control will be carried out. And in this case, when the actual arrival time of delayed vehicle is within the threshold, the waiting vehicle should de-part from the station immediately after all the passengers have finished their transfer and do not need to 
wait until the threshold; when the actual arrival time of delayed vehicle is beyond the threshold, the waiting vehicle should depart from the station after waiting for a holding time threshold.

Analysis of holding control adaptability. Not all the holding control at the transfer station can achieve an obvious effect, so the adaptability of holding control is analyzed. The effect of holding control is related to the situation of bus arrival, the departure interval of bus routes, the transfer passenger volume at the station and so on. Therefore, the object of study is defined in order to gain remarkable results through holding control. About the situation of bus arrival, the case where the origin route (OR) is late arriving and the destination route (DR) is punctually arriving at the station is the focus in study. In regard to the departure interval of bus routes, the case where DR has long interval and OR has short or long interval is studied. Regarding the transfer passenger volume, the stations which has greater transfer passenger volume is considered.

Frame work of dynamic holding control. The basic framework of holding control for the transfer station is established, as is shown in figure 1. When the DR vehicle is detected into the transfer station, there are two cases as follows. Case 1: the DR vehicle is already late, then it has to depart from the station immediately after the passengers are set down and picked up. Case 2: the DR vehicle is arriving at the station ahead of time or on time and the OR vehicle do not arrive punctually, then the passengers on the bus and passengers getting on the bus at this station are counted. Meanwhile the passengers at the downstream station and passengers transfer-ring from OR vehicle are predicted. Then the holding time threshold will be calculated. When the arrival time of delayed OR vehicle is predicted, the decision of holding control or not can be made by comparing the threshold with the arrival time.

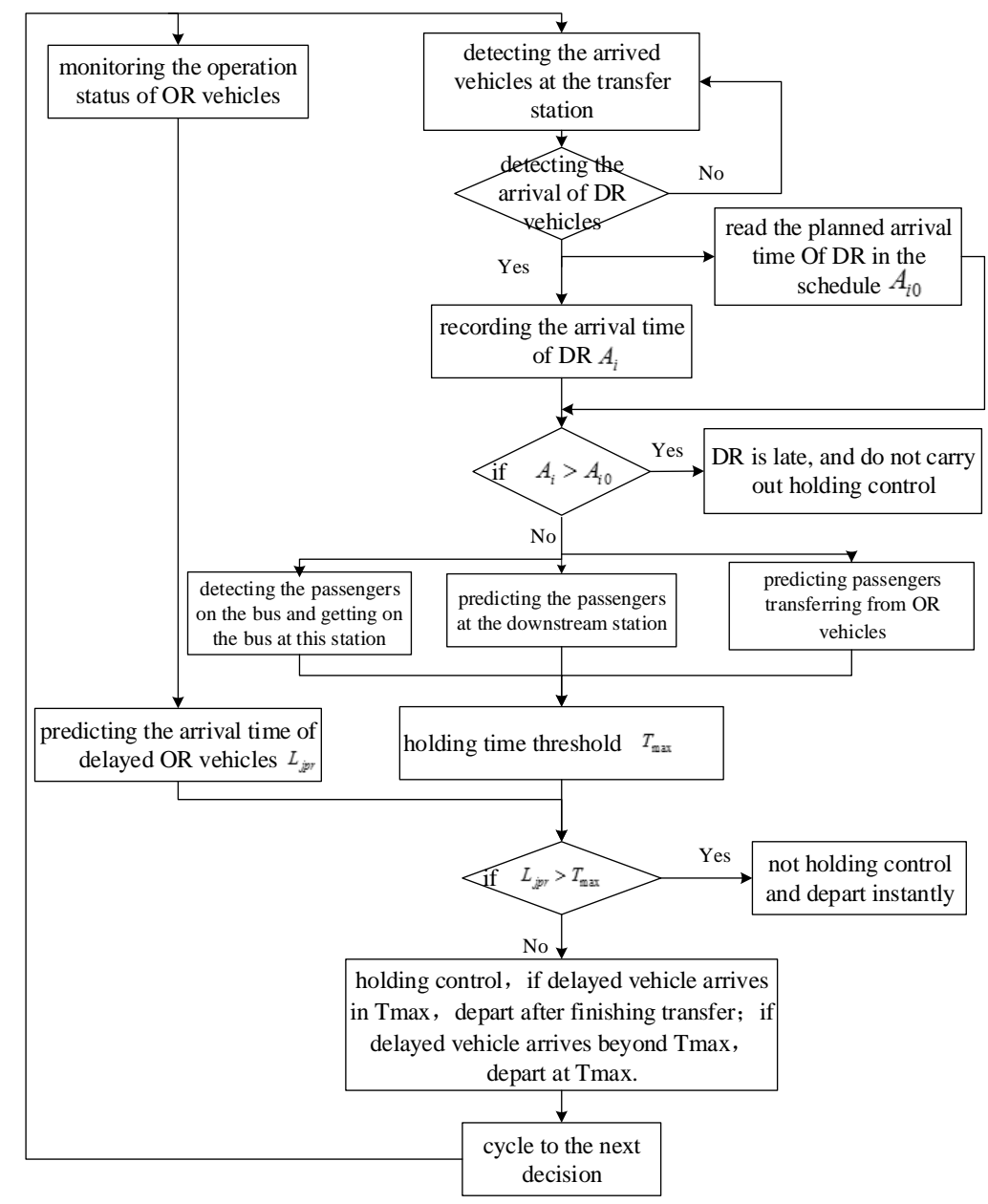

Figure.1 The Framework of Dynamic Holding Control 


\section{Model of Dynamic Holding Control}

Assumptions. The proposed model is established by considering the characteristics of both OR and DR, and based on assumptions as follows.

(1) DR vehicles travel following the planned schedule;

(2) Buses can't depart from the station ahead of schedule;

(3) The transfer passengers from OR to DR and the passengers at the downstream station are known;

(4) Intervals of OR and DR are coordinated and DR interval is an integral multiple of OR interval;

(5) The research period is defined as DR interval $\mathrm{H}$ (the longer interval);

(6) Passengers are arriving at the station at a constant rate;

(7) Arrival time of delayed OR vehicles can be predicted and delayed time will not exceed the interval.

Total social costs. The change of total social costs consists of two parts: change of operation costs and change of passengers' costs. The change of operation costs is caused by the extra waiting time due to the holding control. The change of passengers' costs is caused by passengers related to OR and DR, including passengers on the bus, passengers getting on the bus at current and downstream stations, passengers who are planned to wait for the next bus but get on this bus due to the holding control, and passengers who succeed in transferring due to the holding control.

(1) Change of operation costs (DR) caused by the holding control

$$
C_{0}=t_{h} \llbracket c_{0}
$$

where, ${ }^{t_{h}}$ is the holding time of DR vehicle (minute); $c_{0}$ is the operation costs per vehicle per unit time (RMB/minute).

(2) Change of passengers' costs

(a) An increased cost of waiting time for passengers on the bus (DR) due to the holding control

$$
C_{P}^{I N V}=P_{i, k}^{I N K} \square t_{h} \llbracket c_{p}
$$

where, $P_{i, k}^{i N K}$ is passengers still on the bus $\mathrm{i}$ at the station $\mathrm{k}$ (person); ${ }^{c_{p}}$ is the value of unit time for passengers (RMB/minute/person).

(b) An increased cost of waiting time for passengers waiting for this vehicle (DR) due to the holding control $C_{P}^{A R 1}=H \sqcap P_{\text {Arate }} \llbracket t_{h} \llbracket c_{p}$

where, $H$ is the interval of DR vehicles (minute); $P_{\text {Arate }}$ is the arriving rate of passengers waiting for DR (person/minute).

(c) A reduced cost of waiting time for passengers who are planned to wait for the next bus but get on this bus due to the holding control.

$$
C_{P}^{A R 2}=-P_{\text {Arate }}\left\lceil t_{h} \llbracket\left(H-t_{h}\right) \llbracket c_{p}\right.
$$

(d) An increased cost of waiting time for passengers waiting for this bus at the downstream station due to the holding control

$$
C_{P}^{D O W N}=P_{i}^{D} \llbracket t_{h} \llbracket c_{p}
$$

where, $P_{i}^{D}$ is the number of passengers waiting for this DR vehicle at the downstream station (person).

(e) A reduced cost of waiting time for passengers succeeding in transferring due to the holding control

$$
C_{P}^{T R}=-P_{j, i}^{T R} \llbracket\left(H-L_{j}\right) \llbracket c_{p} \llbracket \varphi\left(t_{h}-L_{j}\right)
$$

where, 
$\varphi\left(t_{h}-L_{j}\right)= \begin{cases}0 & t_{h}<L_{j} \\ 1 & t_{h} \geq L_{j}\end{cases}$

where, $P_{j, i}^{T R}$ is number of passengers transferring from OR to DR (person); $L_{j}$ is the delayed time according to the schedule (minute).

Model. The change of total social costs in this paper include the change of operation costs and the change of all the relevant passengers' costs. Then, the model is de-fined by the holding time.

$$
\begin{aligned}
& \min C_{H}\left(t_{h}\right)=C_{0}+C_{P}^{I N V}+C_{P}^{A R 1}+C_{P}^{A R 2}+C_{P}^{D O W N}+C_{P}^{T R} \\
& =t_{h} \llbracket c_{0}+P_{i, k}^{I N K} \llbracket t_{h} \llbracket c_{p}+H \square P_{\text {Arate }} \llbracket t_{h} \llbracket c_{p}-P_{\text {Arate }} \square t_{h} \llbracket\left(H-t_{h}\right) \llbracket c_{p} \\
& +P_{i}^{D} \llbracket t_{h} \llbracket c_{p}-P_{j, i}^{T R} \llbracket\left(H-L_{j}\right) \llbracket c_{p} \llbracket \rho\left(t_{h}-L_{j}\right) \\
& \text { s.t. }\left\{\begin{array}{c}
0<t_{h}<H \\
P_{i, k}^{I N K}+P_{\text {Arate }} \square\left(H+t_{h}\right)<\alpha V \text { cap }
\end{array}\right.
\end{aligned}
$$

Where,

$\varphi\left(t_{h}-L_{j}\right)= \begin{cases}0 & t_{h}<L_{j} \\ 1 & t_{h} \geq L_{j}\end{cases}$

In the actual situation, the arrival time of delayed vehicles is unable to be accurately predicted. So, when the delayed time is unknown, a searching method through matlab should be applied in solving this model and a holding time threshold will be obtained. Then, the decision of holding control can be made by comparing the holding time threshold and delayed time. The application of holding time threshold can reduce the influence caused by the prediction error and improve the probability of successful transfer.

\section{Case Study}

There is a transfer at the station $\mathrm{k}$ from OR to DR. The interval of OR is 10 minutes and the interval of DR is 20 minutes. The operation costs per vehicle per unit time of buses are $5 \mathrm{RMB}$ and the value of unit time for passengers is 0.6 RMB. Now the bus i of DR arrive at the station $\mathrm{k}$ on time and the bus $\mathrm{j}$ of OR which has a transfer with bus $i$ is late. At the station $k$, the number of passengers on bus $i$ is 30 and the arrival rate of passengers waiting for bus $i$ is 0.5 person/minute. As is predicted, the number of passengers waiting for the bus $i$ at the downstream station is 20 , and the number of passengers transferring from bus $j$ to bus $i$ is 10 . And the capacity of the bus is 80 persons/vehicle. Considering the requirement of passengers' comfort, load factor is set within 0.9. According to the model above, objective function is obtained:

$$
\begin{aligned}
& \min C_{H}\left(t_{h}\right)=C_{0}+C_{P}^{I N V}+C_{P}^{A R 1}+C_{P}^{A R 2}+C_{P}^{D O W N}+C_{P}^{T R} \\
& =5 \mathrm{t}_{h}+18 \mathrm{t}_{h}+6 \mathrm{t}_{h}-0.3 \mathrm{t}_{h}\left(20-\mathrm{t}_{h}\right)+12 \mathrm{t}_{h}-6\left(20-L_{j}\right) \varphi\left(t_{h}-L_{j}\right) \\
& \text { s.t. }\left\{\begin{array}{c}
0<t_{h}<20 \\
30+0.5 \square\left(20+t_{h}\right)<0.9 * 80
\end{array}\right.
\end{aligned}
$$

where, 
$\varphi\left(t_{h}-L_{j}\right)= \begin{cases}0 & t_{h}<L_{j} \\ 1 & t_{h} \geq L_{j}\end{cases}$

The results are calculated by matlab as shown in table 1 .

Table 1. the optimal holding time and change of total social costs in different delay time

\begin{tabular}{|c|c|c|c|c|c|c|c|c|c|c|c|}
\hline $\begin{array}{c}\text { Delay time } \\
\text { [min] }\end{array}$ & 0 & 0.5 & 1 & 1.5 & 2 & 2.5 & 3 & 3.5 & 4 & 4.5 & 5 \\
\hline $\begin{array}{c}\text { Optimal } \\
\text { holding } \\
\text { time[min] }\end{array}$ & 0 & 0.5 & 1 & 1.5 & 2 & 2.5 & 0 & 0 & 0 & 0 & 0 \\
\hline $\begin{array}{c}\text { Change of } \\
\text { total social } \\
\text { costs[RMB] }\end{array}$ & -120 & -99.4 & -78.5 & -57.4 & -36 & -14.4 & 0 & 0 & 0 & 0 & 0 \\
\hline
\end{tabular}

We can know from the results above that the holding time threshold $\mathrm{Tmax}=2.5 \mathrm{~min}$. When the delay time of bus $\mathrm{j}$ is predicted as Ljpr, the decision of holding control or not can be made.

(1) If Ljpr $<=2.5 \mathrm{~min}$, holding;

(2) If Ljpr $>2.5 \mathrm{~min}$, not holding and departing immediately.

\section{Conclusion}

The paper proposed a dynamic holding control strategy based on the traditional holding control. The adaptability of the holding control is analyzed and the research objective is defined as follows: DR arrives at the transfer station on time and the interval of DR is longer while OR is late. And the framework of dynamic holding control is put forward. The various factors influencing the holding control are studied in this paper. Then the model of dynamic holding control is established so that the holding control threshold can be calculated. Finally, a case is studied in applying the model of dynamic holding control.

For research of dynamic holding control at the transfer station for buses, there still exist many problems. More researches have to be concentrated on the prediction of bus arrival time and passenger flow volume. Besides, the transfer between multiple bus routes should be studied further. So that the dynamic holding control can be applied in the actual operation better and improve the quality and attractiveness of public transport service.

\section{References}

[1] Xiucheng Guo, Yadan Yan, Operation Reliability Ananlysis and Scheduling Control of Bus Transit, Nanjing, Southeast University Press, 2013.

[2] Dessouky M, Hall R, Nowroozi A, et al. Bus dispatching at timed transfer transit stations using bus tracking technology, Transportation Research Part C 7(1999) 187-208.

[3] Hall R, Dessouky M, Lu Q. Optimal holding times at transfer stations, Computers \& Industrial Engineering 40(2001) 379-397.

[4] Cluett C, Jenq J H. Utah Transit Authority's Connection Protection System, Perceptions of Riders and Operators, Journal of Public Transportation 8(2005):73-87.

[5] Teng, J. Theory and method of bus coordination dispatching at the transit hub [D], 2005. 
[6] Teng, J, Yang, X.G. Coordination optimization for bus holding at the transfer hub, System Engineering Theory and Practice 28(2008) 156-163.

[7] Dessouky M, Hall R, Zhang L, et al. Real-time control of buses for schedule coordination at a terminal, Transportation Research Part A, Policy and Practice 37 (2003) 145-164.

[8] Chowdhury M S. Intermodal transit system coordination with dynamic vehicle dispatching. ProQuest, UMI Dissertations Publishing, 2000.

[9] Chung E. Transfer coordination model and real-time strategy for inter-modal transit services. ProQuest, UMI Dissertations Publishing, 2009.

[10] Chung E H, Shalaby A. Development of control strategy for intermodal connection protection of timed-transfer transit routes, Transportation Research Record, Journal of the Transportation Research Board (2006) 3-10. 